KURZ GEMELDET

Allergy News

\section{Viele Ekzeme für wenig Lohn}

W er sein Geld mit Putzen verdient, setzt sich für einen Stundenlohn von 7-11 Euro einem hohen Risiko aus. Nach Klinikdaten von 803 Reinigungsfrauen litten 81,6\% an Handekzemen, $31 \%$ an allergischer Kontaktdermatitis und $19,4 \%$ an atopischer Dermatitis. Im Patchtest reagierten sie auf Gummizusätze wie die Erstarrugsbeschleuniger Thiuram $(11,6 \%)$, Dithiocarbamate $(3,4 \%)$ und Mercaptobenzothiazole (1,8\%). Weitere Allergene waren Desinfektionsmittel wie Formaldehyd (3,4\%), Benzalkoniumchlorid $(3,5 \%)$ und Glyoxal $(3,7 \%)$.

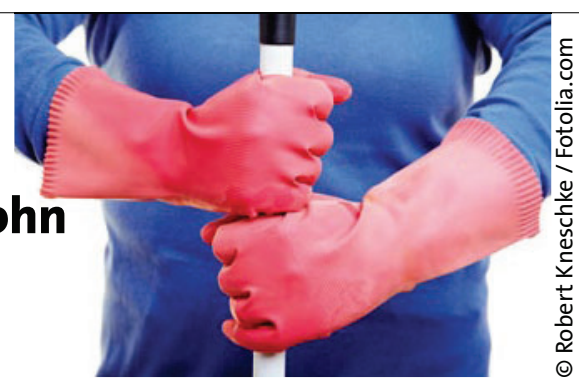

Ein Problem ist das Tragen von Einmalhandschuhen. Diese setzen größere Mengen an Gummizusätzen frei als wiederverwendbare und sind weniger chemikalienresistent. Empfohlen werden dicke wiederverwendbare Schutzhandschuhe mit Baumwollfutter. Dr. Robert Bublak

Liskowsky J et al. Contact Dermatitis 2011; 65: 159-66

\title{
Asthmakranke sprechen auch auf Plazebo an
}

D ie Selbsteinschätzung von Patienten und das Ergebnis der Lungenfunktion können beim Asthma bronchiale deutlich voneinander abweichen. Das belegt eine Studie mit 46 leicht bis mittelschwer Erkrankten, in der das Betamimetikum Salbutamol gegen zwei Plazebotherapien sowie Nichtbehandeln getestet wurde. Die FEV nahm unter Salbutamol um $20 \%$ und damit etwa dreimal so stark zu wie unter Plazebo oder ohne Behand- lung. Die subjektive Verbesserung lag unter Salbutamol bei 50\%, erreichte aber mit der Plazeboinhalation oder der Scheinakupunktur ebenfalls $45 \%$ oder $46 \%$. Ärzte müssen die subjektive Verbesserung des Asthmas daher mit Vorsicht interpretieren. Die Wahrnehmung des Patienten ist aber wichtig. Dr. Beate Schumacher

Wechsler M et al. N Engl J Med 2011; 365: 119-26

\section{Das Geheimnis des Apfel(sinen)kerns}

$\mathrm{D}$ rei Kinder stellten australische Allergologen vor ein Rätsel. Ein sechsjähriges Mädchen, das seinen Fruchtsalat nicht vertragen hatte, wurde in der Klinik vorgestellt. Ein Hautpricktest auf das Fruchtfleisch von Orangen fiel negativ aus, doch bei der oralen Provokation reagierte das Kind schon auf einen einzigen Orangenkern anaphylaktisch. Vergleichbar lag der Fall bei einer Fünfjährigen, die eine Anaphylaxie erlebte als sie ein durchgeschnittenes Stück Orange aß. Das dritte Kind war ein 15 Monate alter Junge. Er schluckte zwei Teelöffel industriell hergestelltes Apfelmus für Babys und benötigte Minuten später drei Adrenalininjektionen. Dabei hatte er Äpfel und hausgemachtes Mus zuvor toleriert. Der Pricktest auf frischen Apfel war tatsächlich negativ, positiv verlief er auf die käufliche Babykost und auf Apfelkerne. Die Krankenakten wiesen alle drei Kinder als sensibel gegen Erd- und andere Nüsse aus. Mit Nüssen war die Babykost nach Analysen des Herstellers nicht kontamininert; dafür bestätigte er, dass die Äpfel vor der Verarbeitung nicht entkernt werden. Die Allergolgen vermuten eine Verbindung zwischen Allergien gegen die beim Aufschneiden oder Zerbeißen exponierten Fruchtkerne und gegen Nüsse. Dr. Dana Weiß Aug 1; DOI: 10.1016/j.jaci.2011.07.005

\section{BTA trocknet Fließnasen}

otulinumtoxin A (BTA) hat in einer Pilotstudie mit fünf Patienten die Symptome der idiopathischen Rhinitis deutlich gebessert. HNO-Ärzte aus München setzten den Probanden zwei Spritzen mit 200 Einheiten/ml submukoperichondral zu beiden Seiten ins anteriore nasale Septum. Während der folgenden zwei Wochen sank der tägliche Verbrauch der Patienten an Taschentüchern von durchschnittlich 21 auf 5,8. Bei allen Patienten besserte sich die nasale Obstruktion. $\mathrm{Ne}$ benwirkungen traten nicht auf. Gegenüber der bekannten Injektionen von BTA in die Nasenmuscheln ist die septale Technik leichter auszuführen und birgt eine geringere Gefahr intravaskulärer Applikationen.

Dr. Robert Bublak

Braun T et al. Am J Otolaryngol 2011 Mar 18; DOI: 10.1016/j.amjoto.2011.01.004

\section{Asthma durch E-Smog?}

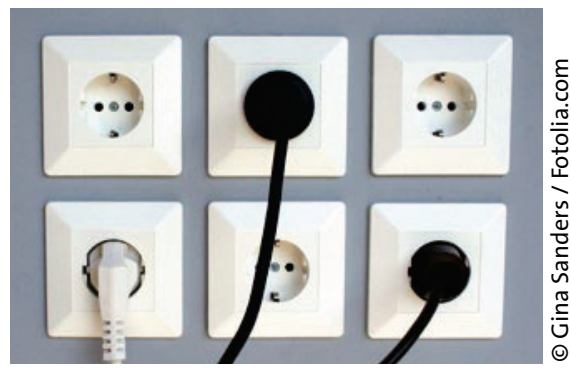

ind Mütter während der Schwangerschaft Elektrosmog ausgesetzt, steigt mit der Dosis auch das Risiko ihrer Kinder, später an Asthma zu erkranken. US-Forscher bestimmten die mediane 24-Stunden-Magnetfeldexposition von 626 Schwangeren und vermerkten über 13 Jahre hinweg Asthmadiagnosen ihrer Sprösslinge. Die Analyse ergab, dass sich die Asthmarate pro Anstieg der magnetischen Flussdichte um 0,1 Mikrotesla $(\mu \mathrm{T})$ linear um 15\% erhöhte. Bei Kindern von Frauen mit der höchsten Belastung $(>0,2 \mu \mathrm{T})$ lag die Asthmarate 3,5-fach höher als bei Kindern von Frauen mit der geringsten Belastung $(\leq 0,03 \mu \mathrm{T})$. Dr. Robert Bublak

Li DK et al. Arch Pediatr Adolesc Med 2011 Aug 1; DOI: 10.1001/archpediatrics.2011.135 